\title{
Interaction of the C-terminal tail region of the metabotropic glutamate receptor 7 with the protein kinase C substrate PICK1
}

\author{
Oussama El Far, ${ }^{1}$ José Airas, ${ }^{1}$ Erhardt Wischmeyer, ${ }^{2}$ Ralf B. Nehring, ${ }^{2}$ Andreas Karschin ${ }^{2}$ and Heinrich Betz ${ }^{1}$ \\ ${ }^{1}$ Department of Neurochemistry, Max-Planck-Institute for Brain Research, Deutschordenstrasse 46, 60528 Frankfurt-am-Main, \\ Germany \\ ${ }^{2}$ Molecular Neurobiology of Signal Transduction, Max-Planck-Institute for Biophysical Chemistry, 37070 Göttingen, Germany
}

Keywords: mGluR, PDZ domain, rat, yeast two-hybrid

\begin{abstract}
Group III metabotropic glutamate receptors (mGluRs) are highly enriched in the presynaptic terminals of glutamatergic synapses where they mediate feedback inhibition of neurotransmitter release. Here, we used the yeast two-hybrid system to identify a direct interaction of the C-terminal tail region of mGluR7 with the rat homologue of the protein kinase C substrate PICK1. This interaction is specifically mediated by the very C-terminal amino acids of the receptor and can be reconstituted in human embryonic kidney 293 cells by transfection of full-length mGluR7 and PICK1 cDNAs. Quantitative $\beta$-galactosidase assays revealed that among the different group III mGluRs, mGluR7 is the major PICK1 binding partner although other subfamily members can also interact with PICK1. These data indicate that PDZ domain-containing proteins might contribute to the presynaptic localization of group III mGluRs.
\end{abstract}

\section{Introduction}

Glutamate serves as a neurotransmitter at the vast majority of excitatory synapses in the mammalian CNS. The postsynaptic actions of glutamate are mediated by two receptor families, the 'ionotropic' glutamate receptors (GluRs), which form glutamate-gated cation channels (Hollmann \& Heinemann, 1994) and the 'metabotropic' glutamate receptors (mGluRs), which are coupled to different effector enzymes via distinct G-proteins (Conn \& Pin, 1997). Presynaptically, mGluRs mediate feedback inhibition of transmitter release by downregulating the voltage-activated $\mathrm{Ca}^{2+}$ channels that trigger synaptic vesicle exocytosis (Pin \& Bockaert, 1995; Pin \& Duvoisin, 1995; Herlitze et al., 1996; Ikeda, 1996; Takahashi et al., 1996).

At present, eight different members of the mGluR family have been identified which, based on sequence homologies, pharmacological properties and intracellular transduction pathways, are classified into three distinct groups. Groups I and II include mGluRs that are predominantly postsynaptic, whereas group III encompasses receptors (mGluRs 4, 7 and 8) that are principally presynaptically localized (Shigemoto et al., 1997). In particular, mGluR7 was found to be highly enriched at active zones in terminals of hippocampal pyramidal cells (Shigemoto et al., 1996). This selective localization and its low glutamate affinity have led to the hypothesis that mGluR7 functions as a low-pass filter that inhibits synapses which are firing above certain frequencies (Shigemoto et al., 1996). Consistent with this view, mGluR7 knockout mice show epileptic seizures at $>12$ weeks of age in addition to an impairment of amygdala-dependent averse responses (Masugi et al., 1999).

Correspondence: Dr Heinrich Betz, as above.

E-mail: betz@mpih-frankfurt.mpg.de

Received 30 June 2000, revised 25 August 2000, accepted 18 September 2000
The molecular mechanisms that underlie the highly restricted localization of mGluR7 at active zones are unknown. Their elucidation may provide important insights into how a neuron localizes receptor proteins at neurotransmitter release sites. Recently, Stowell \& Craig (1999) have shown that the C-terminal tail region of mGluR7 is important for the axonal targeting of this receptor. In an attempt to isolate gene products that may contribute to the selective presynaptic localization of group III mGluRs, we recently employed affinity purification to identify intracellular binding partners of mGluR7A, one of the two known splice variants A and B in the mGluR7 tail region (Flor et al., 1997; O'Connor et al., 1999). This approach failed to identify proteins involved in the targeting of this receptor to active zones but disclosed calmodulin as an essential coregulator of glutamatergic presynaptic autoinhibition (O’Connor et al., 1999). Here, we used the yeast two-hybrid system to search for additional proteins which bind to the C-terminal tail of mGluR7 and report that PICK1 (Protein Interacting with $C$ Kinase), a PDZ (for PSD 95, Dlg1 and ZO-1) domain containing protein that interacts with activated protein kinase C (PKC) alpha (Staudinger et al., 1995) and AMPAtype ionotropic glutamate receptors (Xia et al., 1999), binds specifically to the extreme C-terminal tail sequence of mGluR7. As PICK1 has been shown to be enriched at synapses (Xia et al., 1999), this interaction may be essential for the selective localization of mGluR7 at the active zones of glutamatergic nerve terminals.

\section{Materials and methods}

\section{Yeast two-hybrid analysis}

Fragments encoding the complete $\mathrm{C}$-terminal tail regions of mGluR7A, 7B, 4A, 4B, 8A and $8 \mathrm{~B}$ were generated by PCR as described (O'Connor et al., 1999), inserted into the inducible pGilda 
bait vector and used with the DupLex-A Two-Hybrid system kit (OriGene Technologies, Inc., Rockville, USA) in binary interaction tests. The bait vector containing the mGluR7A C-terminal tail fragment was used to screen $10^{6}$ independent recombinant clones of a rat brain cDNA library in pJG4-5 using the supplied yeast strain EGY 48. Out of 129 blue colonies picked from the original galactoseinduced selection plates (-Ura, -Trp, -Leu, -His), only one contained a plasmid that allowed specific and reproducible growth on selective medium as well as $\beta$-galactosidase induction upon isolation and retransformation with the bait vector. This clone contained an inframe insertion of the full-length rat homologue of mouse PICK1 (Staudinger et al., 1995) with an additional $5^{\prime}$ untranslated sequence of $111 \mathrm{bp}$ lacking any stop codon (sequence deposited under GenBank accession no. AJ240083). Beta-galactosidase activities were quantitatively determined using o-nitrophenyl $\beta$-D-galactopyranoside according to the Yeast Protocols Handbook from Clontech.

\section{Expression and mutant constructs}

A myc-tag was N-terminally introduced into the rat PICK1 cDNA after the first methionine codon by performing PCR on the isolated pJG4-5-PICK1 clone with an EcoR1-flanked myc-tag-containing sense primer and an antisense primer encompassing the internal Apa1 site located $120 \mathrm{bp}$ after the start codon. Using the introduced EcoR1 site and the internal Sal1 site of pJG4-5 at position 2630, the myctagged PICK1 cDNA was subcloned into pEGFP-c2 (Clontech) to generate a EGFP-myc-PICK1 construct. Mutant glutathione transferase (GST)-mGluR7A fusions (O'Connor et al., 1999) and pGildamGluR7A tail constructs (mGluR7A-LVI/AAA), in which the three $\mathrm{C}$-terminal amino acids were replaced by alanines, were generated by PCR using the pGilda-mGluR7A tail construct as template. GFP fused to the PDZ domain of PICK1 (EGFP-myc-PICK ${ }_{1-113}$ ) was generated by PCR using primers with flanking EcoR1 and Sal1 restriction sites; after excision, the product was ligated into the pEGFP-c2 vector.

To inactivate the PICK1 PDZ domain, the lysine and aspartic acid residues at positions 27 and 28 located in the carboxylate binding loop where replaced by alanines (EGFP-myc-PICK $1-113$ KD/AA) (Staudinger et al., 1997). This construct was generated by PCR using two complementary 29mer mutant primers encompassing the mutated region in combination with respective sense and antisense primers to generate two overlapping PCR products that covered the entire 1-113 PICK1 N-terminal amino acid sequence. The resulting PCR products were then mixed and used as template in a new PCR reaction with the same sense and antisense primers that were used to generate the wild-type mycPICK $_{1-113}$ cDNA. The resulting EcoR1 and Sal1 flanked PCR product was subcloned into pEGFP-c2 (EGFP-myc-PICK ${ }_{1-113}$ ).

All constructs were verified using automated DNA sequencing.

\section{Transfection experiments}

The EGFP-myc-PICK1 cDNA was transfected into human embryonic kidney (HEK) 293 cells (ATCC no: CRL-1573) by calcium phosphate coprecipitation (Wigler et al., 1977). After $48 \mathrm{~h}$, the cells from four $10-\mathrm{cm}$ culture dishes were collected and solubilized for $1 \mathrm{~h}$ in $1.5 \mathrm{~mL}$ binding buffer (BB; $50 \mathrm{mM}$ Tris- $\mathrm{Cl} \mathrm{pH} \mathrm{7.4,} 50 \mathrm{mM}$ $\mathrm{NaCI}$, protease inhibitor cocktail Complete ${ }^{\mathrm{TM}}$ from BoehringerMannheim) containing 2\% (w/v) Triton X-100. The solubilized material was collected after centrifugation at $100000 \mathrm{~g}$ at $4{ }^{\circ} \mathrm{C}$ for $1 \mathrm{~h}$ and used immediately for affinity adsorption on glutathioneSepharose preloaded with GST or GST-wild-type and mutant mGluR7A tail fusion proteins.

\section{Bacterial expression and binding studies}

Expression of GST fusion proteins in E. coli BL21 (Stratagene, La Jolla, CA, USA) was induced by adding $0.5 \mathrm{~mm}$ isopropyl $\beta$-Dthiogalactopyranoside for 3-5 h. After passage through a French press, the $100000 \mathrm{~g}$ supernatants containing soluble GST-fusion protein were kept frozen $\left(-76^{\circ} \mathrm{C}\right)$ until needed. Soluble fractions $(0.25 \mathrm{mg}$ of total bacterial proteins) were incubated with $50 \mu \mathrm{L}$ of glutathione-Sepharose beads for $2 \mathrm{~h}$ at $4{ }^{\circ} \mathrm{C}$ followed by three washes, each, in $1 \mathrm{~mL}$ of $\mathrm{BB}, \mathrm{BB}$ containing $0.1 \%$ (w/v) Triton $\mathrm{X}$ 100 , and $\mathrm{BB}$ containing $2 \mathrm{mM} \mathrm{MgCl}_{2}$ and $0.5 \mathrm{~mm}$ ATP. The immobilized fusion proteins were then incubated with $3 \mathrm{~mL}$ of cleared two-fold diluted detergent extract of transfected HEK 293 cells at $4{ }^{\circ} \mathrm{C}$ for $4-5 \mathrm{~h}$. After agitated incubation at $4{ }^{\circ} \mathrm{C}$ for another 4-5 h and four consecutive washes with BB containing $0.2 \%(\mathrm{w} / \mathrm{v})$ Triton X-100, bound proteins were eluted with SDS sample buffer as described (O'Connor et al., 1999). The unbound material was precipitated with trichloroacetic acid [final concentration $7.2 \%(\mathrm{w} / \mathrm{v})$ ] and resuspended in SDS sample buffer. Samples were resolved by $10 \%$ SDS polyacrylamide gel electrophoresis and analysed by Western blotting using either the 9E10 antimyc monoclonal antibody (Evan et al., 1985) or the 'Living Colors ${ }^{\mathrm{TM}}$, rabbit anti-GFP polyclonal antibodies (Clontech, Heidelberg, Germany).

For binding experiments with native PICK1, a $1.5 \%$ (w/v) Triton $\mathrm{X}-100$ extract of a rat brain homogenate (corresponding to $1 \mathrm{mg}$ of total protein) in HB buffer (HB: $20 \mathrm{~mm}$ Hepes, pH 7.4, $100 \mathrm{~mm}$ $\mathrm{NaCl}, 100 \mu \mathrm{M}$ ATP, protease inhibitor cocktail Complete ${ }^{\mathrm{TM}}$ ) was incubated in a total volume of $3.3 \mathrm{~mL}$ with $0.5 \mathrm{mg}$ of immobilized GST, GST-mGluR7A or GST-mGluR7A-LVI-AAA prewashed and equilibrated in $\mathrm{HB}$. After overnight incubation at $4{ }^{\circ} \mathrm{C}$ followed by four consecutive washes with $\mathrm{HB}$ containing $0.2 \%$ (w/v) Triton $\mathrm{X}$ 100 , bound proteins were eluted with SDS sample buffer as described. For visualizing native PICK1 on Western Blots, a rabbit polyclonal antipeptide antibody was generated (Cocalico Biologicals, Inc., Reamstown/PA, USA) by immunization with the N-terminal PICK1 sequence FADLDYDIEEDKLGIPTVPGK conjugated to keyhole limpet haemocyanin. Specific antipeptide antibodies were isolated by affinity purification on a peptide-conjugated matrix following published procedures (Harlow \& Lane, 1988).

\section{Electrophysiological recordings}

cRNAs encoding concatenated pairs of G-protein-activated inwardly rectifying channels Kir 3.1/3.2 (Wischmeyer et al., 1997), full-length mGluR7A and PICK1 were coinjected into Xenopus oocytes. Gprotein $\beta \gamma$ subunit regulation of the rectifying $\mathrm{K}^{+}$currents was then measured according to published procedures (Wischmeyer et al., 1997) in $96 \mathrm{~mm}$ external $\mathrm{K}^{+}$at $-80 \mathrm{mV}$ in the presence of $100 \mu \mathrm{M}$ of the group III mGluR selective agonist L(+)-2-amino-4-phosphonobutyric acid (L-AP4).

\section{Immunocytochemistry}

HEK 293 cells grown on fibronectin-coated cover slips were transfected with the indicated expression constructs. Forty-eight hours after transfection, cells were washed with phosphate buffered saline (PBS) and fixed with ice-cold 4\% (w/v) paraformaldehyde in PBS at room temperature for $5 \mathrm{~min}$. Using PBS containing 5\% (w/v) bovine serum albumin and $0.25 \%(\mathrm{w} / \mathrm{v})$ Triton X-100 as staining buffer, the anti-Flag mouse monoclonal M2 (Kodak Scientific) antibody [to detect Flag-mGluR7A and Flag-mGluR7A(LVI-AAA)] and the Living colours ${ }^{\mathrm{TM}}$ rabbit anti-GFP polyclonal antibodies (Clontech) were utilized as primary antibodies at $1: 1000$ and $1: 200$ dilutions, respectively. After washing, Alexa-conjugated 
A
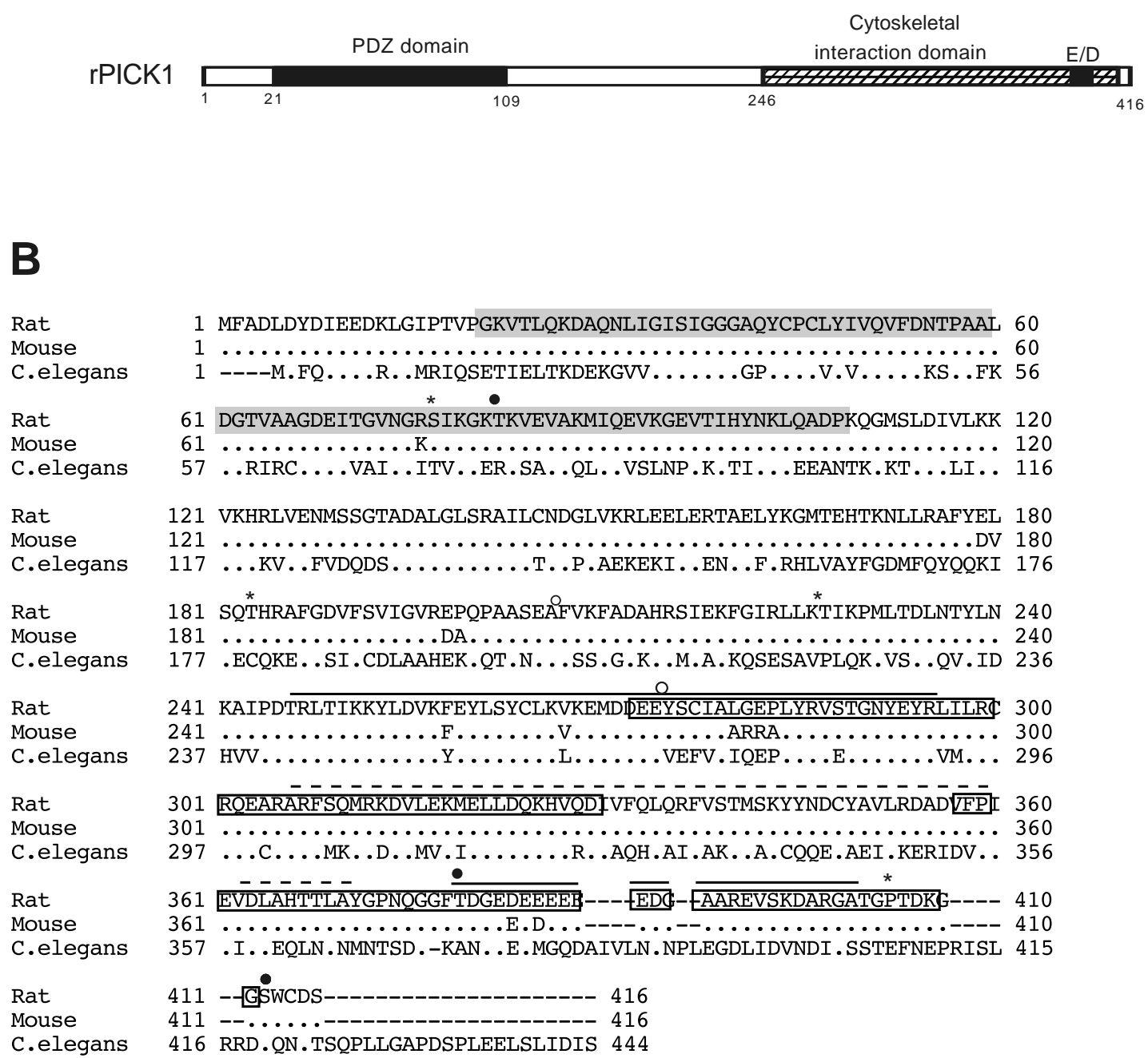

FIG. 1. Domain structure of PICK1. (A) Schematic representation of the identified domains on PICK1. (B) Alignment of rat, mouse and C. elegans PICK1 protein sequences. The shadowed sequence represents the PDZ domain. A neurofilament homology region identified by BLAST search is boxed, and MAP1B and cytokeratin 21 homology domains are overligned with a solid and a dashed line, respectively. Putative PKC, tyrosine kinase and Casein kinase phosphorylation sites are indicated by asterisks, and open and closed circles, respectively. E/D indicates a glutamate- and aspartate-rich region.

secondary antibodies (Jackson ImmunoResearch Laboratories, PA, USA) were employed to differentially visualize the primary antibodies. Confocal pictures were taken using a Leica TCS-SP confocal microscope, equipped with Leica TCS-NT 16551 software.

\section{Results}

In an attempt to identify intracellular proteins that bind to mGluR7 and thus may contribute to its specific presynaptic localization, we used the C-terminal tail region (amino acids 850-915) of the mGluR7A splice variant in a yeast two-hybrid screen of an adult rat brain cDNA library. From $10^{6}$ colonies screened, 129 were isolated from selection plates. Sequencing identified one clone encoding the full-length rat PICK1 cDNA. PICK1 is a known PDZ domaincontaining protein that has been shown to bind the activated form of PKC alpha (Staudinger et al., 1995, 1997) and the AMPA receptor subunits GluR2 and GluR3 (Xia et al., 1999). The clone isolated here contained an in-frame N-terminal untranslated sequence devoid of stop codons which extends the mouse PICK1 sequence (Staudinger et al., 1995) by 40 amino acids. The rat PICK1 sequence displays identities of 43, 96 and $97 \%$ with the respective C. elegans, mouse and human homologues (Fig. 1). All PICK1 proteins contain a single N-terminal PDZ domain (aa 21-104 in rat PICK1). In mammalian PICK1, a consensus sequence for an ATP/GTP binding loop (P loop; aa $75-82$ in rat PICK1) is found between the predicted $\beta E$ strand and helix B of the PICK1 PDZ domain (Daniels et al., 1998). The Cterminal half of the protein displays overlapping homology domains to the microtubule-associated protein MAP1B, neurofilament $\mathrm{L}$ and cytokeratin 21 (Fig. 1). Throughout the rat PICK1 sequence, five putative PKC phosphorylation sites (positions 77, 183, 227, 249, 407), one tyrosine phosphorylation site (275) and three casein kinase II phosphorylation consensus sites (amino acids 82, 379, 412) were found (Fig. 1).

To biochemically confirm the interaction of PICK1 with the mGluR7A C-terminal tail, we overexpressed myc-tagged PICK1 fused to enhanced green fluorescent protein (EGFP-myc-PICK1) in HEK 293 cells. A Triton X-100 extract of the transfected cells was 

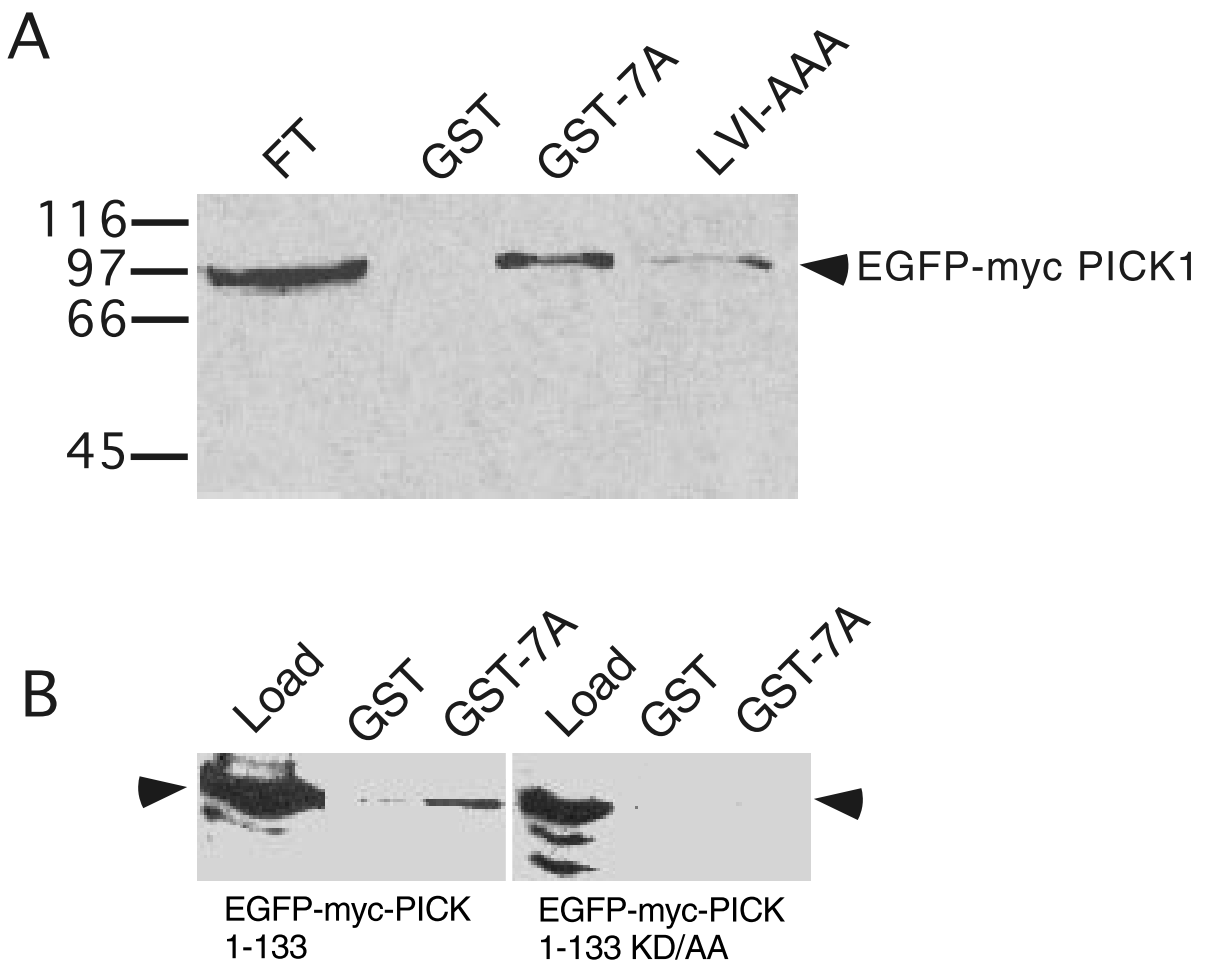

FIG. 2. In vitro binding of the C-terminal tail of mGluR7A to PICK1. GST and GST fusion proteins of the C-terminal tail of mGluR7A and the mutant tail mGluR7A(LVI-AAA) were immobilized on glutathione-Sepharose beads and incubated with Triton X-100 extracts obtained from the following sources: (A) HEK 293 cells expressing EGFP-myc-PICK1; (B) HEK 293 cells expressing EGFP-myc-PICK 1 133 or EGFP-myc-PICK ${ }_{1-133}$ KD/AA; (C) adult rat brain homogenate. After removing nonspecifically bound material by washing, proteins were eluted with SDS sample buffer and analysed by Western blotting using the following antibodies: (A) antimyc 9E10; (B) anti-GFP; and (C) affinity-purified anti-PICK1. Note that only the wild-type mGluR7A tail (A) and PICK1 PDZ domain (B) constructs were able to interact. Also, in rat brain extracts PICK1 was barely detected under the conditions used for the Western blot shown in $C$, indicating a high enrichment of PICK1 by the immobilized mGluR7A tail. Asterisks in C indicate nonspecifically labelled bands; FT; flow through.

then utilized to perform a pull-down assay using either GST or GSTmGluR7A immobilized on glutathione-Sepharose beads. EGFP-mycPICK1 bound only to GST-mGluR7A but not to GST (Fig. 2A). This confirms the yeast two-hybrid results and shows that, in vitro, the Cterminal tail of mGluR7A binds to PICK1.

Different studies have shown that PDZ domains bind to C-terminal amino acids of interacting proteins (Kornau et al., 1997; Songyang et al., 1997; Daniels et al., 1998). Modification of the C-terminal sequence in PDZ domain binding proteins is known to decrease or abolish protein interaction. To test whether the very C-terminal residues of the mGluR7A tail are important for its binding to PICK1, we replaced the last three amino acids (LVI) by alanines. This mutation drastically decreased the binding of PICK1 to the mGluR7A tail, as judged by quantitative two-hybrid analysis (Fig. 3). When examining the binding of the immobilized GST-mGluR7A-LVI-AAA tail to EGFP-myc-PICK1 overexpressed in HEK 293 cells, binding was similarly strongly reduced as compared to wild-type GSTmGluR7A (Fig. 2A). These results indicate that mGluR7A Cterminal binding to PICK1 is governed by conventional PDZ domain properties.

To demonstrate that the interaction of PICK1 with the mGluR7A tail is mediated by the unique PDZ domain of PICK1, we fused either the wild-type or a mutated PDZ domain of PICK1 (EGFP-myc-
PICK $_{1-133}$ and EGFP-myc-PICK $1-133$ KD/AA, respectively) to GFP. Substitution of residues K27 and D28 by alanines has been previously shown to inactivate the binding of PKC to PICK1's PDZ domain (Staudinger et al., 1997). When using these constructs in pull-down assays, only EGFP-myc-PICK $1-133$ but not EGFP-myc-PICK $1-133$ KD/AA bound the mGluR7A tail (Fig. 2B). These results show that lysine 27 and aspartate 28 in the carboxylate binding loop of the PDZ domain of PICK1 are essential for binding to mGluR7A.

To confirm that native PICK1 interacts with the mGluR7A tail, we also performed binding assays with a Triton X-100 extract from rat brain as source of PICK1. As shown in Fig. 2C, incubation of the extract with the wild-type GST-mGluR7A tail construct but not GST or GST-mGluR7A-LVI-AAA led to a high enrichment of brain PICK1. These data indicate that native PICK1 binds with high affinity to the extreme C-terminal sequence of mGluR7A.

To examine whether PICK1 interacts also with other group III mGluRs, and whether alternative splicing (Flor et al., 1997; O'Connor et al., 1999) might modify PICK1 interactions, we also tested the mGluR 8A, 8B, 4A, 4B and 7B (Conn \& Pin, 1997) tail domains in binary two-hybrid assays (Fig. 3). Of the group III mGluR cytoplasmic domains tested, only mGluR4B failed to display detectable binding to PICK1. The mGluR7B splice variant also showed very strong PICK1 binding. Similarly, the mGluR 4A, 8A 
and $8 \mathrm{~B}$ tail domains were also found to bind PICK1 with, however, at least 20-fold reduced efficacy as compared to mGluR7A (Fig. 3). Thus, most presynaptic mGluR family members may be able to bind
A

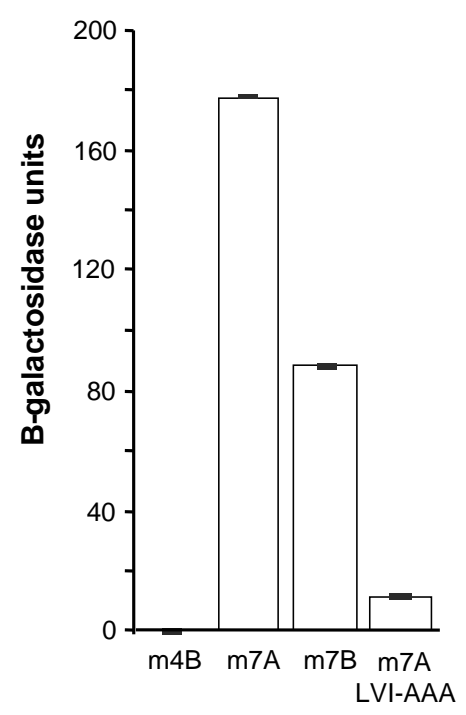

B

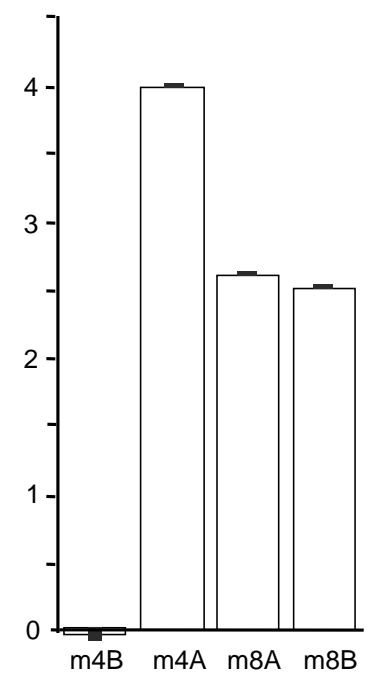

FIG. 3. Comparative quantification of the two-hybrid interactions between group III mGluR C-termianl tails and PICK1. The liquid phase assay of the two-hybrid system was used to quantify and compare the relative strength of interactions between PICK1 and mGluR 4A, 4B, 7A, 7B, 8A, 8B. The results are expressed in relative beta-galactosidase units. (A) Comparison of the strength of mGluR 4B, 7A, 7B and m7A-LVI/AAA C-terminal tail interactions with PICK1. (B) As in A, but for mGluR 4B, 4A, 8A, 8B Cterminal tails. Note the scale difference between $\mathrm{A}$ and $\mathrm{B}$. this PKC substrate. However, by far the strongest interactions were seen with mGluR7A and B.

To examine whether the interaction of PICK1 with mGluR7 affects receptor signalling (O'Connor et al., 1999), we monitored $\mathrm{K}^{+}$currents in oocytes coinjected with cRNAs encoding concatenated G-protein activated inwardly rectifying potassium curents (Kir) 3.1/3.2 channels (Wischmeyer et al., 1997) and full-length mGluR7A in the presence or absence of the PICK1. Activation of G-protein signalling through mGluR7A was induced by $100 \mu \mathrm{M}$ of the agonist L-AP4. No significant differences in the resulting $\mathrm{K}^{+}$currents were noted upon coexpression of PICK1 (current values of $-5.15 \pm 1.5 \mu \mathrm{A}$ in control and $-5.35 \pm 1.9 \mu \mathrm{A}$; in PICK1-expressing cells; $n=5$, each). This result shows that, in contrast to the regulation of mGluR7A driven Gprotein signalling by calmodulin (O'Connor et al., 1999), PICK1 does not seem to participate directly in the regulation of effector protein coupling.

It has recently been shown that in cultured hippocampal neurons PICK1 staining overlaps with synaptic markers, such as glutamic acid decarboxylase and synaptophysin, and is enriched at synapses formed along neuronal processes (Torres et al., 1998; Xia et al., 1999). We therefore investigated the interaction of PICK1 and mGluR7 in a cellular context using heterologous expression in HEK 293 cells. Wild-type or mutant mGluR7A cDNAs and PICK1 PDZ domain fusions with EGFP were cotransfected, and protein distributions were monitored by fluorescence and immunostaining. Upon single transfection, expression of the wild-type Flag-mGluR7A and the mutant Flag-mGluR7A-LVI/AAA receptors resulted in prominent plasma membrane staining (Fig. 4A and B). In contrast, both the wild-type and mutated PICK1 constructs (EGFP-myc-PICK 1-133 $_{13}$ and EGFP-myc-PICK ${ }_{1-133} \mathrm{KD} / \mathrm{AA}$, respectively) produced a diffuse cytoplasmic fluorescence that sometimes showed small cytoplasmic
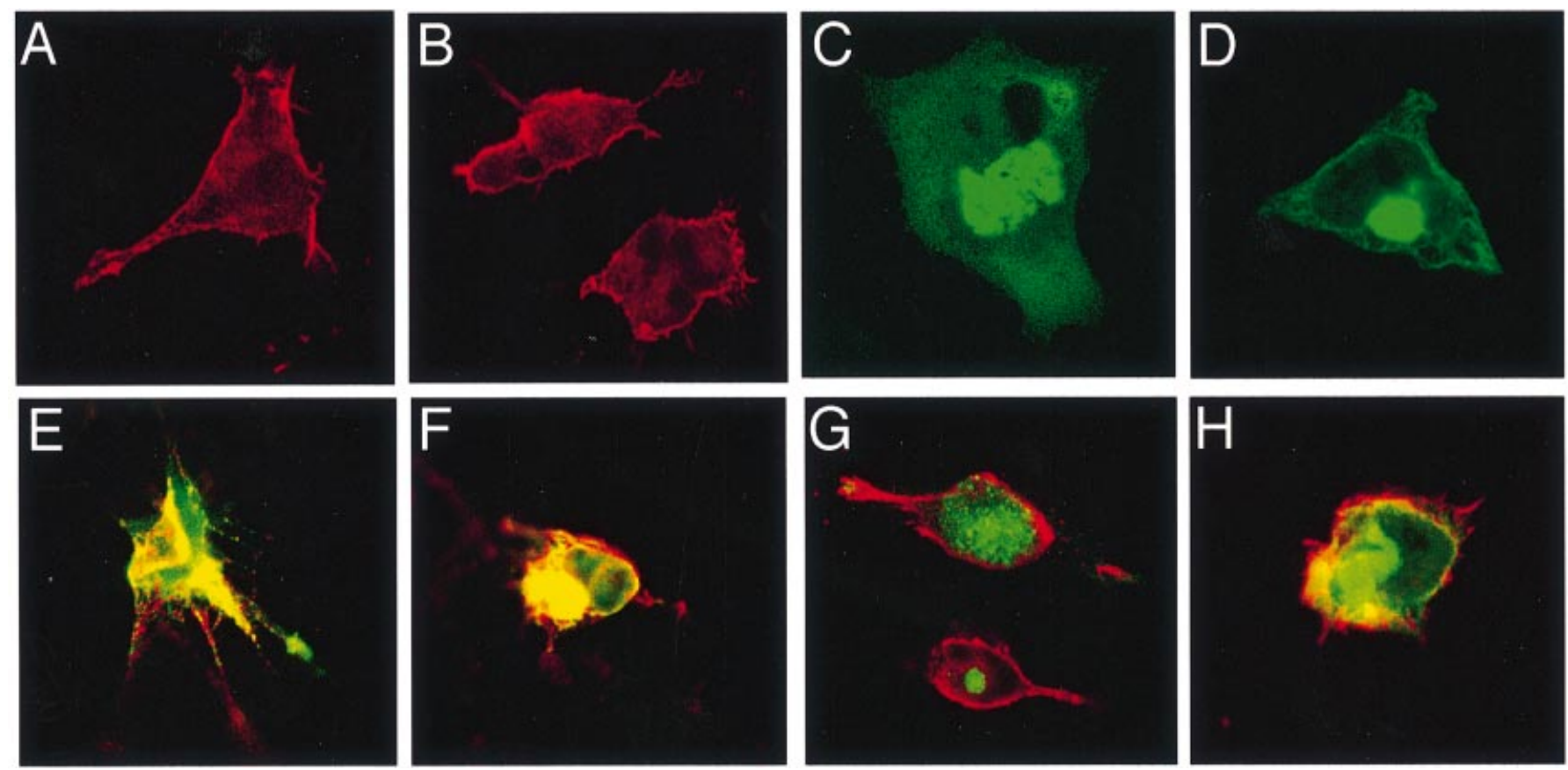

FIG. 4. Colocalization of mGluR7A and PICK1 in transfected cells. HEK 293 cells were transfected with the following constructs: (A) Flag-mGluR7A; (B) Flag-mGluR7A-LVI/AAA; (C) EGFP-myc-PICK $1-133$; (D) EGFP-myc-PICK ${ }_{1-133}$ KD/AA; (E,F), Flag-mGluR7A + EGFP-myc-PICK $1-133$; (G) Flag-mGluR7A + EGFP-myc-PICK ${ }_{1-133}$ KD/AA; $(\mathrm{H})$ Flag-mGluR7A-LVI/AAA + EGFP-myc-PICK ${ }_{1-133}$. Cells were fixed and stained with anti-GFP, anti-Flag or a mixture of both antibodies, as described under Material and Methods. Immunostainings are shown in green for GFP fusion proteins and in red for the Flag-tagged receptor. For costainings, only overlays of the green and red channels are shown. Note that mGluR7 and PICK1 colocalize completely in E and F, partially in $\mathrm{H}$ but not at all in $\mathrm{G}$. 
clusters and, more frequently, larger intracellular blobs (Fig. 4C and D). The latter occasionally resembled the previously described perinuclear staining of PICK1 in transfected cells (Staudinger et al., 1995). Coexpression of the wild-type PICK1 PDZ domain and mGluR7A fusion constructs resulted in significant colocalization of both proteins at the plasma membrane as well as, in some cells, an accumulation of mGluR7A immunoreactivity at perinuclear blobs of PICK1 as described above (Fig. 4E and F). As expected, the EGFPmyc-PICK $_{1-133}$ KD/AA fusion construct failed to show any colocalization with the wild-type Flag-mGluR7A protein, confirming that mGluR7 binding depends on the integrity of the PDZ domain (Fig. 4G). Similarly, the Flag-mGluR7A-LVI/AAA mutant receptor that displayed reduced binding to the EGFP-myc-PICK1 construct in biochemical experiments (Fig. 2) showed only weak colocalization with the wild-type PDZ domain of EGFP-myc-PICK1 $1_{1-133}$ (Fig. $4 \mathrm{H}$ ). These results prove that the mGluR7A-PICK1 interaction found in vitro can be faithfully reproduced upon cotransfection in mammalian cells.

\section{Discussion}

In this study, we have shown that the protein kinase $\mathrm{C}$ substrate PICK1 interacts with the major cytoplasmic domain of mGluR7A. We discovered this interaction in a yeast two-hybrid screen that led to the isolation of rat PICK1. PICK1 binding is mediated by the extreme $\mathrm{C}$-terminus of the receptor, as replacement of the last three amino acids leucine, valine and isoleucine of mGluR7A by alanines decreased the intensity of the interaction by about 18-fold as quantified in yeast. In addition, binding of a recombinant GSTmGluR-7A tail fusion protein to native PICK1 or EGFP-myc-PICK1 in vitro was significantly reduced when introducing the triple-alanine mutation. Similarly, the integrity of the PICK1 PDZ domain proved crucial for this interaction. Substitution of residues K27 and D28 in the carboxylate binding loop of PICK1 by alanines is known to abolish PKC binding (Staudinger et al., 1997) and also prevented its interaction with mGluR7A in both binding and cotransfection experiments. Attempts to coimmunoprecipitate mGluR7A and PICK1 from and to colocalize these proteins in neuronal tissues were not successful, due to the properties of the available PICK1 and mGluR7 antibodies (O. El Far, unpublished data). However, PICK1 binding to mGluR7A in a cellular context could be clearly confirmed in cotransfection experiments, in which full-length mGluR7A was found to bind a fusion protein harbouring both GFP and the PDZ domain of PICK1. Again, this cellular interaction required the Cterminal amino acids of the receptor.

The interaction of mGluR7A with PICK1 is based on a conventional PDZ domain-C-terminal peptide interaction. It should be noted, however, that within the mGluR family, the interaction was not specific for mGluR7A. Besides mGluR7B, mGluR4A, 8A and 8B tails also bound PICK1 although with reduced affinity. In contrast, the structurally divergent mGluR4B tail showed no detectable binding. We therefore conclude that PICK1 could have a more general role in presynaptic mGluR localization or function. This is consistent with immunocytochemical data on cultured hippocampal neurons which indicate a strong enrichment of PICK1 at sites of synaptic contact (Xia et al., 1999). Whether PICK1 is present pre- and/or postsynaptically will have to be clarified by ultrastructural studies.

While our study was in progress, PICK1, in addition to binding PKC alpha and AMPA receptors, was found also to interact with ephrins and class I ADP ribosylation factors (ARFs). Thus, 15 different proteins and/or splice variants possessing rather divergent
TABLE 1. Proteins tested for binding to PICK1

\begin{tabular}{lll}
\hline Protein & $\begin{array}{l}\text { C-terminal amino } \\
\text { acid sequence }\end{array}$ & $\begin{array}{l}\text { Interaction* } \\
\text { with PICK1 }\end{array}$ \\
\hline mGluR4A & TYTNHAI & + \\
mGluR4B & WVFGDGL & - \\
mGluR7A & SYNNLVI & + \\
mGluR7B & YTIPPTV & + \\
mGluR8A & SYSDHSI & + \\
mGluR8B & VKSGSTS & + \\
GluR2 $\dagger$ & GIESVKI & + \\
GluR3 $\dagger$ & GTESVKI & + \\
GluR4 $\dagger$ & VIASDLP & + \\
GluR4C $\dagger$ & GTESIKI & + \\
PKCalpha & PILQSAV & + \\
EphB2§ & QIQSVEV & + \\
EphA7§ & HGTGIQV & + \\
ephrin-B1§ & ANIYYKV & + \\
rMuSK§ & AEGTVGV & + \\
ARF19 & NQLKNKK & + \\
ARF3I & NQLRNQK & + \\
\hline
\end{tabular}

The last seven $\mathrm{C}$-terminal amino acids of the proteins listed are shown. *The ' + ' indicates a positive interaction with PICK, and the '-' a failure to detect a significant signal. Additional data are from: $†$ Xia et al., 1999, Staudinger et al., 1997, §Torres et al., 1998 and "T Takeya et al. 2000.

C-terminal sequences have now been reported to bind PICK1 (summarized in Table 1). These C-terminal sequences are characterized by the presence of either hydrophobic or hydrophilic basic residues at both the 0 and the -2 positions. The PDZ domain in PICK1 thus appears to tolerate significant variations in the C-terminal binding sequence of its partner proteins, although with important consequences for the interaction strength. Most strikingly, the mGluR7B fusion protein that carries the proline-rich sequence PPTV as the terminal peptide also bound avidly to PICK1. In addition, the very different $\mathrm{C}$-terminal amino acids of PKC alpha, a receptor tyrosine kinase of the ephrin family and ephrin ligands, the skeletal muscle receptor tyrosine kinase MuSK, the ionotropic glutamate receptor subunits GluR2, 3 and 4 (Xia et al., 1999) as well as the class I ARFs 1 and 3 all interact tightly with the unique PDZ domain of PICK1 (Table 1).

Based on binding specificities and sequence homologies, PDZ domains fall into two classes. Class I sequences select for peptides with the C-terminal consensus motif [E-(S/T)-X-(V/I)], whilst class II domains favour peptides with hydrophobic or aromatic side chains at the three carboxyterminal residues (consensus [F/Y-X-F/V/A]). Class II PDZ domains differ from class I by the presence of a secondary binding pocket that can accommodate a hydrophobic residue at position -2 (Songyang et al., 1997; Daniels et al., 1998). When considering these characteristics and comparing the primary sequence of the PICK1 PDZ domain with those of different members of class I and II PDZ motifs as well as with the terminal sequences of the known PICK1 binding partners, it is difficult to classify the PICK1 PDZ domain into class I or II subtypes. We therefore propose that this PDZ region specifies a new class III of PDZ domains that tolerate hydrophobic and basic but not acidic residues at position -2 .

Despite the large number of proteins shown to be able to bind to PICK1 in vitro, the in vivo role of these interactions is far from being clear. It is possible that the strength of PICK1 binding to different binding partners is regulated by additional factors, e.g. PICK1 phosphorylation (Staudinger et al., 1995), oligomerization (Staudinger et al., 1997) and/or additional binding proteins. In any 
case, the interaction with mGluR7A described here is of high affinity, suggesting that this membrane protein may be one of PICK1's principal presynaptic binding partners.

Recently, we have shown that the N-terminal region of mGluR7 tail binds in a mutually exclusive manner both $\mathrm{Ca}^{2+} /$ calmodulin $(\mathrm{CaM})$ and the $\beta \gamma$ subunits of trimeric G-proteins (O'Connor et al., 1999). The interaction of $\mathrm{Ca}^{2+} / \mathrm{CaM}$ with the receptor is crucial for its signalling function and has been proposed to drive the release of bound beta-gamma subunits from the tail upon $\mathrm{Ca}^{2+}$ influx (O'Connor et al., 1999). It has also been shown that the site of CaM binding is phosphorylated by PKC (Nakajima et al., 1999; J. Airas et al., unpublished data). As phosphorylation of the CaM binding site of different proteins is known to regulate CaM binding (Graff et al., 1989; Adarem, 1992; Minakami et al., 1997), and because PICK1 oligomerizes independently of its PDZ domain (Staudinger et al., 1997), we suggest that PICK1 participates in the formation of a complex that contains mGluR7 and activated PKC alpha under conditions that require mGluR7 to dissociate from CaM. As PICK1 binds only to activated PKC alpha, the formation of such a complex should depend on the activation of PKC alpha through a mechanism that still awaits identification, such as signalling through group I and/ or II mGluRs (Conn \& Pin, 1997). A specific compartmentalization of such a complex might be provided by an association of PICK1 with cytoskeletal elements as suggested by its C-terminal sequence similarities with keratin 21, neurofilament L and MAP-1B. In addition, as coexpression of PICK1 induces the clustering of ephrin receptors, of ephrin ligands and of the postsynaptic AMPA receptor subunits GluR2 and 3, PICK1 may in addition serve as an adaptor protein that organizes the group III mGluRs located at active zones into readily available clusters of higher-order receptor complexes that exert multiple signalling and structural functions.

\section{Acknowledgements}

We thank S. Nakanishi for the mGluR7A cDNA, V. O'Connor and D. Roth for helpful discussions and critical reading of the manuscript, and Anja Niehuis and Bärbel Kohler for technical assistance. This work was supported by the Human Frontier Science Program Organization (fellowship to O.E.F), Deutsche Forschungsgemeinschaft (SFBs 474 and 406) and Fonds der Chemischen Industrie.

\section{Abbreviations}

ARF, ADP ribosylation factor; BB, binding buffer; CaM, calmodulin; GluR, ionotropic glutamate receptor; HEK, human embryonic kidney; Kir, potassium inwardly rectifying; L-AP4, L(+)-2-amino-4-phosphonobutyric acid; mGluRs, metabotropic glutamate receptors; MuSK, skeletal muscle receptor tyrosine kinase; PBS, phosphate buffered saline; PDZ, PSD95, Dlg1, ZO1; PICK1, protein interacting with $\mathrm{C}$ kinase; $\mathrm{PKC}$, protein kinase $\mathrm{C}$.

\section{References}

Adarem, A. (1992) The MARCKS Brothers: a family of protein kinase C substrates. Cell, 71, 713-716.

Conn, P.J. \& Pin, J.P. (1997) Pharmacology and functions of metabotropic glutamate receptors. Annu. Rev. Pharmacol. Toxicol., 37, 205-237.

Daniels, D.L., Cohen, A.R., Anderson, J.M. \& Brunger, A.T. (1998) Crystal structure of the hCASK PDZ domain reveals the structural basis of class II PDZ domain target recognition. Nature Struct. Biol., 5, 317-325.

Evan, G.I., Lewis, G.K., Ramsay, G. \& Bishop, J.M. (1985) Isolation of monoclonal antobodies specific for human c-myc protooncogene product. Mol. Cell. Biol., 5, 3610-3616.

Flor, P.J., Van Der Putten, H., Rüegg, D., Lukic, S., Leonhardt, T., Bence, M., Sansig, G., Knöpfel, T. \& Kuhn, R. (1997) A novel splice variant of a metabotropic glutamate receptor, human mGluR7b. Neuropharmacology, 36, 153-159.

Graff, J.M., Young, T.N., Johnson, J.D. \& Blackshear, P.J. (1989) Phosphorylation-regulated calmodulin binding to a prominent cellular substrate for protein kinase C. J. Biol. Chem., 264, 21818-21823.

Harlow, E. \& Lane, D. (1988) Antibodies. A Laboratory Manual. Cold Spring Harbor Laboratory Press, New York.

Herlitze, S., Garcia, D.E., Mackie, K., Hille, B., Scheuer, T. \& Catterall, W.A. (1996) Modulation of $\mathrm{Ca}^{2+}$ channels by G-protein beta gamma subunits. Nature, 380, 258-262.

Hollmann, M. \& Heinemann, S. (1994) Cloned glutamate receptors. Аnnu. Rev. Neurosci., 17, 31-108.

Ikeda, S.R. (1996) Voltage-dependent modulation of N-type calcium channels by G-protein beta gamma subunits. Nature, 380, 255-258.

Kornau, H.C., Seeburg, P.H. \& Kennedy, M.B. (1997) Interaction of ion channels and receptors with PDZ domain proteins. Curr. Opin. Neurobiol., 7, 368-373.

Masugi, M., Yokoi, M., Shigemoto, R., Muguruma, K., Watanabe, Y., Sansig, G., van der Putten, H. \& Nakanishi, S. (1999) Metabotropic glutamate receptor subtype 7 ablation causes deficit in fear response and conditioned taste aversion. J. Neurosci., 19, 955-963.

Minakami, R., Jinnai, N. \& Sugiyama, H. (1997) Phosphorylation and calmodulin binding of the metabotropic glutamate receptor subtype 5 (mGluR5) are antagonistic in vitro. J. Biol. Chem., 272, 20291-20298.

Nakajima, Y., Yamamoto, T., Nakayama, T. \& Nakanishi, S. (1999) A relationship between protein kinase $\mathrm{C}$ phosphorylation and calmodulin binding to the metabotropic glutamate receptor subtype 7. J. Biol. Chem., 274, $27573-27577$.

O'Connor, V., El Far, O., Bofil-Cardona, E., Nanoff, C., Freissmuth, M., Karschin, A., Airas, J.M., Betz, H. \& Boehm, S. (1999) Calmodulin dependence of presynaptic metabotropic glutamate receptor signaling. Science, 286, 1180-1184.

Pin, J.P. \& Bockaert, J. (1995) Get receptive to metabotropic glutamate receptors. Curr. Opin. Neurobiol., 5, 342-349.

Pin, J.P. \& Duvoisin, R. (1995) The metabotropic glutamate receptors: structure and functions. Neuropharmacology, 34, 1-26.

Shigemoto, R., Kulik, A., Roberts, J.D., Ohishi, H., Nusser, Z., Kaneko, T. \& Somogyi, P. (1996) Target-cell-specific concentration of a metabotropic glutamate receptor in the presynaptic active zone. Nature, 381, 523-525.

Shigemoto, R., Kinoshita, A., Wada, E., Nomura, S., Ohishi, H., Takada, M., Flor, P.J., Neki, A., Abe, T., Nakanishi, S. \& Mizuno, N. (1997) Differential presynaptic localization of metabotropic glutamate receptor subtypes in the rat hippocampus. J. Neurosci., 17, 7503-7522.

Songyang, Z., Fanning, A.S., Fu, C., Xu, J., Marfatia, S.M., Chishti, A.H., Crompton, A., Chan, A.C., Anderson, J.M. \& Cantley, L.C. (1997) Recognition of unique carboxyl-terminal motifs by distinct PDZ domains. Science, 275, 73-77.

Staudinger, J., Zhou, J., Burgess, R., Elledge, S.J. \& Olson, E.N. (1995) PICK1: a perinuclear binding protein and substrate for protein kinase C isolated by the yeast two-hybrid system. J. Cell Biol., 128, 263-271.

Staudinger, J., Lu, J. \& Olson, E.N. (1997) Specific interaction of the PDZ domain protein PICK1 with the $\mathrm{COOH}$ terminus of protein kinase $\mathrm{C}$-alpha. J. Biol. Chem., 272, 32019-32024.

Stowell, J.N. \& Craig, A.M. (1999) Axon/dendrite targeting of metabotropic G glutamate receptors by their cytoplasmic carboxy-terminal domains. Neuron, 22, 525-536.

Takahashi, T., Forsythe, I.D., Tsujimoto, T., Barnes-Davies, M. \& Onodera, K. (1996) Presynaptic calcium current modulation by a metabotropic glutamate receptor. Science, 274, 594-597.

Takeya, R., Takeshige, K. \& Sumimoto, H. (2000) Interaction of the PDZ domain of human PICK1 with class I ADP-ribosylation factors. Biochem. Biophys. Res. Com., 267, 149-155.

Torres, R., Firestein, B.L., Dong, H., Staudinger, J., Olson, E.N., Huganir, R.L., Bredt, D.S., Gale, N.W. \& Yancopoulos, G.D. (1998) PDZ proteins bind, cluster, and synaptically colocalize with eph receptors and their ephrin ligands. Neuron, 21, 1453-1463.

Wigler, M., Silverstein, S., Lee, L.S., Pellicer, A., Cheng, Y.C. \& Axel, R. (1977) Transfer of purified herpes virus thymidine kinase gene to cultured mouse cells. Cell, 11, 223-232.

Wischmeyer, E., Döring, F., Wischmeyer, E., Spauschus, A., Thomzig, A., Veh, R. \& Karschin, A. (1997) Subunit interactions in the assembly of neuronal Kir3.0 inwardly rectifying K ${ }^{+}$channels. Mol. Cell. Neurosci., 9, 194-206.

Xia, J., Zhang, X., Staudinger, J. \& Huganir, R.L. (1999) Clustering of AMPA receptors by the synaptic PDZ domain-containing protein PICK1. Neuron, 22, 179-187. 